\title{
Supporting Information Hydrogel Microparticles as Dynamically Tunable Microlenses
}

Jongseong Kim, Michael J. Serpe, and L. Andrew Lyon*

School of Chemistry and Biochemistry, Georgia Institute of Technology, Atlanta, Georgia

30332-0400 (USA)

\section{Experimental Section.}

Materials. All reagents were purchased from Sigma-Aldrich unless otherwise specified. $\mathrm{N}$ Isopropylacrylamide (NIPAm) was re-crystallized from hexanes (J.T. Baker) prior to use. Acrylic acid (AAc) was distilled under reduced pressure. $N, N^{\prime}$-Methylene(bisacrylamide) (BIS) and ammonium persulfate (APS) were used without further purification. 3-Aminopropyltrimethoxysilane (APTMS) was purchased from United Chemical Technologies Inc. The glass substrates used were 24 x $50 \mathrm{~mm}$ Fisher Finest brand cover glass obtained from Fisher Scientific. 95\% and absolute ethanol was used for various purposes in this investigation. Water was distilled and then deionized (DI) to a resistance of at least 18 $\mathrm{M} \Omega$ (Barnstead Thermolyne E-Pure system) and then filtered through a $0.2 \mu \mathrm{m}$ filter to remove particulate matter. $3 \mathrm{M}$ transparency film for laser printers and a Hewlett Packard LaserJet 4000N printer was used for pattern printing.

Microgel Synthesis. Microgels used here had a molar composition of $89.5 \%$ NIPAm, $0.5 \%$ BIS, and $10 \%$ AAc and were synthesized via aqueous free-radical precipitation polymerization, using $300 \mathrm{mM}$ total monomer concentration. Polymerization was carried out in a three-neck, $200 \mathrm{~mL}$ round-bottom flask to which $100 \mathrm{~mL}$ of filtered, aqueous solution of NIPAm and BIS was added. This mixture was heated to $\sim 70{ }^{\circ} \mathrm{C}$ under a $\mathrm{N}_{2}$ atmosphere and stirred with a magnetic stir bar for $\sim 1 \mathrm{hr}$. After $1 \mathrm{hr}, 200 \mu \mathrm{L}$ of AAc was added to the round-bottom flask to bring the total monomer concentration up to $300 \mathrm{mM}$. The reaction was immediately initiated by injection of $1 \mathrm{~mL}$ of a hot $\left(\sim 70^{\circ} \mathrm{C}\right)$ APS solution $(6.13 \mathrm{mM})$ and kept at $70^{\circ} \mathrm{C}$ for 4 hours under a $\mathrm{N}_{2}$ environment. The particles were purified by dialysis against water for $\sim 2$ weeks with the water being changed twice per day, using $10000 \mathrm{MW}$ cut-off dialysis tubing (VWR).

Microlens Substrate Preparation. Glass substrates were cleaned in an Ar plasma (Harrick Scientific) for $30 \mathrm{~min}$ to remove organics from the substrate surface. The substrates were immersed in an ethanolic (absolute ethanol) 1\% APTMS solution for $\sim 2$ hrs. After $2 \mathrm{hrs}$ the substrates were removed from the APTMS solution and again rinsed copiously with 95\% ethanol. These substrates were stored in 95\% ethanol for no longer than 5 days prior to use. Prior to assembly, these substrates were rinsed with DI water and dried under a stream of $\mathrm{N}_{2}$ gas. The substrate was then exposed to an aqueous $10 \%(\mathrm{v} / \mathrm{v})$ microgel solution at $\mathrm{pH} 6.5$ ( $\sim 0.001 \mathrm{M}$ ionic strength) and allowed to completely dry on the substrate ( $24 \mathrm{hrs})$. The substrate was subsequently immersed in DI water for $2 \mathrm{hrs}$, then rinsed with DI water, and dried with nitrogen gas to leave behind only microgels strongly attached to the substrate by electrostatic interaction. Under these conditions, we find that the glass substrate is largely covered with a close-packed monolayer of particles. The thickness of this film is somewhat less than the swollen particle diameter, as the particles spread and flatten on the substrate, as shown in Figure 1 of the main manuscript. Decreasing the particle concentration results in a sparser coverage. While the details of why ordered monolayers arise from this procedure are still a matter of investigation, it is clear that by correctly by balancing the particle concentration, particle-surface attraction, particle-particle repulsion, and capillary forces from the solvent, ordered arrays can be obtained. Different $\mathrm{pH}$ solutions ( 0.001 M ionic strength) were introduced into the void space of a microlens array/silicone gasket/coverslip sandwich assembly to facilitate optical measurements for exploration into the tunable microlens arrays in response to $\mathrm{pH}$ and temperature.

Microscopy. Optical and electron microscopies were used to confirm microgel shape, size and lensing ability. A Hitachi S800 scanning electron microscope (SEM) was used to determine the shape of microgels attached to the glass substrate in the dried state. SEM images were obtained by attaching a dry microgel decorated glass substrate to a SEM peg and placed in the SEM chamber. The substrate was then imaged with an accelerating voltage of $15 \mathrm{kV}$ with the sample tilted relative to the incoming electrons in order to obtain profile images of the microgels. Brightfield transmission, and differential image contrast 
(DIC) optical microscopies were used to confirm the lensing abilities of the microgels attached to the substrate. An Olympus IX70 inverted microscope equipped with a high numerical aperture, oil immersion 100X objective (N.A.=1.30), a microscope objective heater and a Peltier-based temperature stage was used for imaging the pattern under various conditions. Images were captured using a black and white CCD camera (PixelFly, Cooke Corporation) as a function of $\mathrm{pH}$ and temperature.

\section{Particle Refractive Indices and Dimensions.}

While the absolute determination of substrate-supported particle sizes and refractive indices in aqueous media are not readily obtained, estimates as to these values offer some insight as to the relative lensing power of microgels under different conditions. First, we must address the relative size and shape of the substrate-supported microgels. By analysis of the microscopy images we find the following values for the $\mathrm{pH} /$ temperature-dependent particle diameters $\left(D_{T}(p H)\right)$ :

$D_{25}(3.0)=1.6 \mu \mathrm{m}$

$D_{40}(3.0)=0.8 \mu \mathrm{m}$

$D_{25}(6.5)=2.0 \mu \mathrm{m}$

$D_{48}(6.5)=1.0 \mu \mathrm{m}$

Optical microscopy does not directly provide z-dimension information in this mode, so we can only estimate particle volumes by assuming isotropic swelling of a hemisphere. However, it is likely that the particles are somewhat pinned at the surface and that the degree of xy swelling is smaller than that occurring in the z-dimension. If we further conservatively assume that the $D_{40}(3.0)=0.8 \mu \mathrm{m}$ represents a particle containing only $\sim 10 \%$ water by volume (typical for a deswollen pNIPAm sphere), we can estimate the following water volume percents:

$\%$ Water $_{25}(3.0)=89 \%$

$\%$ Water $_{40}(3.0)=10 \%$

$\%$ Water $_{25}(6.5)=94 \%$

$\%$ Water $_{48}(6.5)=54 \%$

Finally, we can estimate the effective refractive indices by assuming a weighted average of the component refractive indices:

$$
n_{\text {microgel }}=\phi_{\text {water }}\left(n_{\text {water }}\right) \dashv \phi_{\text {polymer }}\left(n_{\text {polymer }}\right)
$$

where $n$ is the refractive index and $\phi$ is the volume fraction of that component. From this simple formalism, we can estimate that:

$n_{25}(3.0)=1.343$

$n_{40}(3.0)=1.420$

$n_{25}(6.5)=1.338$

$n_{48}(6.5)=1.377$

Thus, we see that, as shown in the images, the swollen microgels should be poor lenses due to low index contrast relative to water, and that the lensing should improve during thermal 
deswelling. Furthermore, the refractive index contrast for microgels at $\mathrm{pH} 6.5$ below the phase transition temperature is so close to that of water that little if any lensing should be observed.

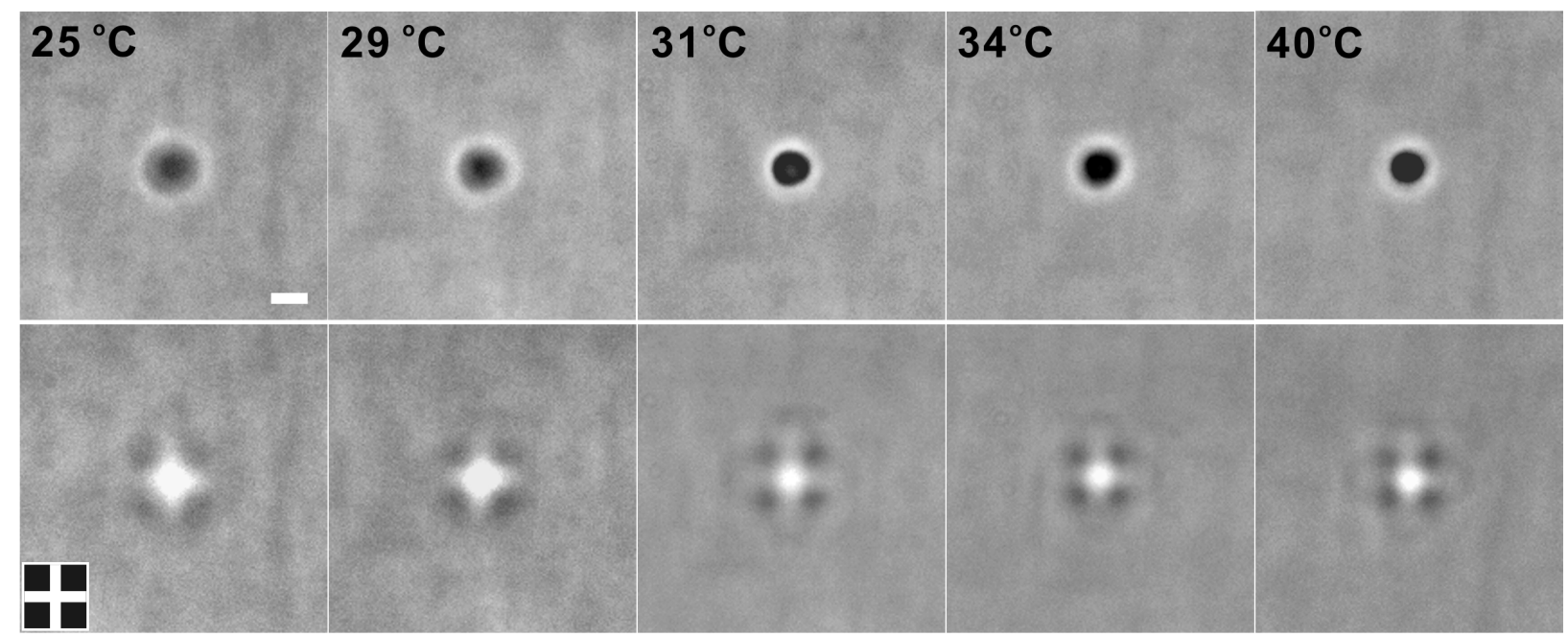

SI Figure 1. Expanded temperature range relative to that shown in Figure 3. (Top) DIC microscopy images of a substrate bound microgel in $\mathrm{pH} 3.0$ solution as a function of temperature. (Bottom) Projection of the cross pattern (inset bottom left) through the microgel at the indicated temperatures. As the microgel size decreases, the focusing ability of the microlens increases. The scale bar is $1 \mu \mathrm{m}$.

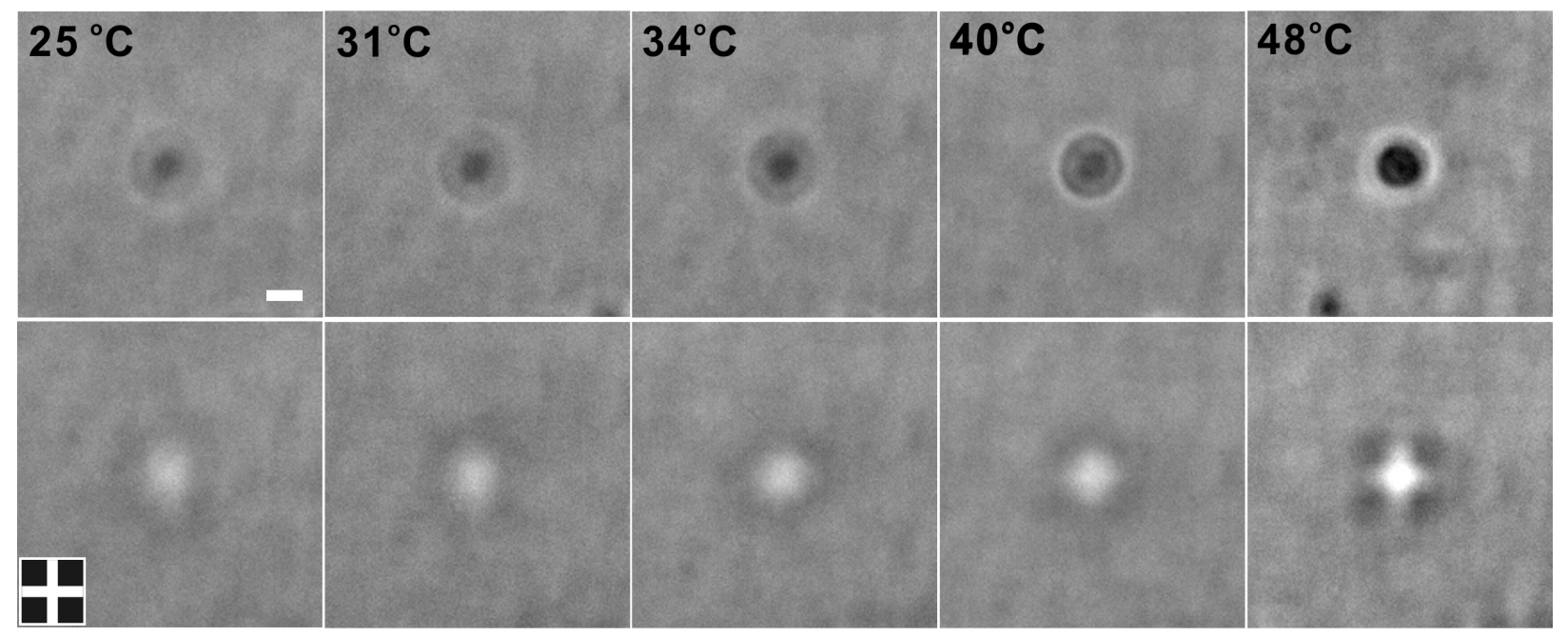

SI Figure 2. Expanded temperature range relative to that shown in Figure 4. (Top) DIC microscopy images of a substrate bound microgel in $\mathrm{pH} 6.5$ solution as a function of temperature. (Bottom) Projection of the cross pattern (inset bottom left) through the microgel at the indicated temperatures. The microgel lensing ability is invariant with temperature over a wide range due to Coulombic repulsion and osmotic pressure in the microgel network as a result of AAc deprotonation. Only upon reaching $48{ }^{\circ} \mathrm{C}$ does the microlens bring the pattern into focus. Note however that the image is still inferior to the pattern projected by the same microlens at $\mathrm{pH}$ 3.0. The scale bar is $1 \mu \mathrm{m}$. 


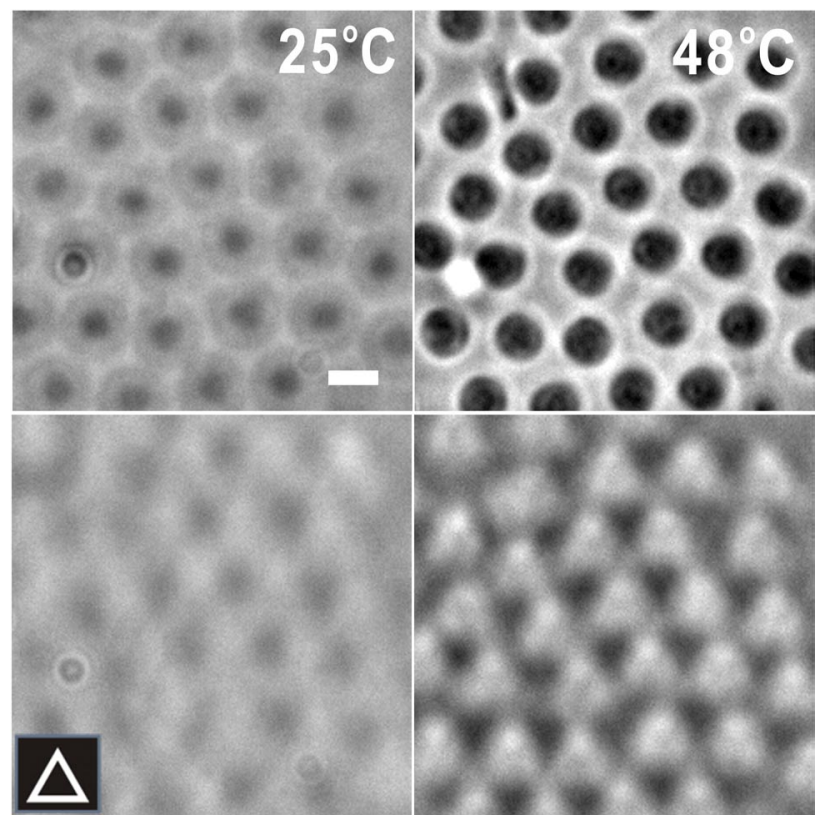

SI Figure 3. DIC and pattern projection images of particle arrays in water at $\mathrm{pH} 6.5$ showing homogeneous image quality from lens to lens. The scale bar is $1 \mu \mathrm{m}$ and the triangle pattern used is shown in the inset. 